\title{
PHASE-DIFFERENCE LOCATION USED TO DETERMINE THE DIRECTION OF ACOUSTIC EMISSION SOURCE
}

\author{
A. A. Popkov \\ Siberian transport university, 191 D. Kovalchuk st., 630049, Novosibirsk, Russian Federation \\ *Corresponding author. Email: zabagy@gmail.com; address for correspondence: ul. D. Kovalchuk 191, 630049, \\ Novosibirsk, Russian Federation. Tel.:8-383-328-03-46
}

The paper describes the usage patterns of closely spaced acoustic emission transducers designed to determine the direction of propagation of acoustic emission impact by calculating the phase delays between the transducers. As a criterion for determining the reliability of the phase delay, correlation analysis is used. The experimental data have established a dependence between the direction of acoustic wave emission and the ratio of phase delays between the transducers.

Keywords: acoustic emission testing, location of emitters, phase-difference, determination of wave direction.

DOI: $10.17804 / 2410-9908.2016 .3 .014-020$

\section{References}

1. Nerazrushayushchiy kontrol: spravochnik [Nondestructive Testing. Reference Book. V.V. Kluev, ed., vol. 7]. M., Mashinostroenie Publ., 2006, 829 p. (In Russian).

2. Stepanova L.N., Lebedev E.Yu., Kabanov S.I., Kanifadin K.V., Ramazanov I.S., Bekher S.A. Acoustic emission testing of the quality of a weld during natural cooling. Kontrol. Diagnostika, 2009, no. 3, pp. 61-67. (In Russian).

3. Stepanova L.N., Bekher S.A., Tenitilov E.S. Methods for the determination of the coordinates of defects in the acoustic emission testing of loose bearing rings. Kontrol. Diagnostika, 2010, no. 4, pp. 61-65. (In Russian).

4. Murav'ev V.V., Bekher S.A., Vlasov K.V. Analysis of Errors in Determining the Coordinates of Acoustic-Emission Sources in Sheet-Steel Structures. Russian Journal of Nondestructive Testing, 2008, vol. 44, iss. 7, pp. 480-485. DOI: 10.1134/S106183090807005X.

5. Nerazrushayushchiy kontrol: spravochnik [Nondestructive Testing. Reference Book. V.V. Kluev, ed., vol. 3]. M., Mashinostroenie Publ., 2006, 864 p. (In Russian). 
Подана в журнал: 23.12 .2015

УДК 534.6:534.88:620.111.1

DOI: $10.17804 / 2410-9908.2016 .3 .014-020$

\title{
ИСПОЛЬЗОВАНИЕ ФАЗОВОЙ ЛОКАЦИИ ДЛЯ ОПРЕДЕЛЕНИЯ НАПРАВЛЕНИЯ ИСТОЧНИКА АКУСТИЧЕСКОЙ ЭМИССИИ
}

\author{
А. А. Попков \\ ФГБОУ ВПО «Сибирский государственный университет путей сообщения», ул. Дуси Ковальчук, 191, \\ 2. Новосибирск, Россия \\ Ответственный автор. Электронная почта: zabagy@gmail.com; Адрес для переписки: 630049, Россия, \\ г. Новосибирск, ул. Дуси Ковальчук, 191; Телефон: +7 (383) 328-03-46
}

В работе представлено описание модели использования близкорасположенных преобразователей акустической эмиссии для определения направления распространения акустикоэмиссионного воздействия путем вычисления фазовых задержек между преобразователями. В качестве критерия достоверности определения фазовой задержки использовался корреляционный анализ. Полученные экспериментальные данные позволили установить зависимость между направлением акустико-эмиссионной волны и отношением фазовых задержек между преобразователями.

Ключевые слова: акустическая эмиссия, локаџия источников акустической эмиссии, фазовая задержка, определение направления источника акустической эмиссии.

\section{1. Введение}

Неопределенность локации источников акустической эмиссии напрямую влияет на достоверность результатов акустико-эмиссионного контроля [1]. Современные критерии оценки степени опасности дефектов требуют применения кластеризации, в том числе с использованием данных о координатах источников акустической эмиссии. Для большинства реальных объектов характерно наличие помех - дискретных сигналов акустической эмиссии, не связанных с развитием усталостных дефектов [2]. Источниками таких сигналов могут являться допустимые дефекты, например растрескивание защитных покрытий, коррозионные повреждения, трение, внешние воздействия. Для исключения помех используется пространственная фильтрация, от правильного применения которой зависит достоверность результатов контроля.

В настоящее время для определения координат источников дискретной акустической эмиссии широко применяются методы [3], основанные на измерении разностей времен прихода сигналов на разнесенные на поверхности объекта контроля преобразователи (акустическая антенна). Методы имеют ограничения [4], связанные со значительным влиянием неопределенности измерения времени начала импульса и скорости его распространения на достоверность координат источников акустической эмиссии при контроле протяженных объектов и объектов сложной формы.

Для повышения точности локации дискретной акустической эмиссии перспективно использовать методы, основанные на регистрации фазы волны в системе близкорасположенных преобразователей (антенные или фазовые решетки) [5]. В отличие от временных методов, фазовый метод в меньшей степени зависит от неопределенности скорости распространения волнового пакета (акустического импульса).

Цель работы - определение зависимости фазовых задержек между близкорасположенными (расстояние меньше длины волны для несущей частоты сигнала) преобразователями от направления распространения акустико-эмиссионного воздействия. 


\section{2. Моделирование}

Исследуемая модель включает в себя три пьезоэлектрических преобразователя, расположенные в вершинах равностороннего треугольника (рис. 1). Для обеспечения однозначности результатов фазового метода локации длина стороны треугольника ограничена длиной волны $\lambda$ акустико-эмиссионного импульса. Регистрация акустических сигналов преобразователями осуществляется разными каналами акустико-эмиссионной системы.

В модели рассматривались источники, расположенные на расстояниях, существенно больших расстояния между преобразователями, поэтому кривизной фронта волны допустимо пренебречь. Все дальнейшие расчеты справедливы только для волн, фронт которых приближенно описывается плоскостью.

В процессе распространения волны на преобразователях возникает разность фаз, которая зависит от направления распространения волны (рис. 1). Фазы регистрируемых сигналов преобразователями 1,2 и 3 рассчитываются по формуле $\varphi_{i}=\frac{2 \pi}{T} t_{i}+\varphi_{i_{0}}$, где $i-$ номер преобразователя.

Разность фаз между сигналами рассчитывается по следующим формулам:

$$
\begin{aligned}
& \Delta \varphi_{1}=\varphi_{3}-\varphi_{2}, \\
& \Delta \varphi_{2}=\varphi_{1}-\varphi_{3} .
\end{aligned}
$$

Волна распространяется между преобразователями 1 и 3 по лучу волны $B M$, между преобразователя 3 и 2 - по лучу $N A$ (рис. 2). Так как $B M$ и $A N$ - кратчайшие расстояния между фронтами волны 1,2 и 3 , то $A N$ и $B M$ перпендикулярны прямой 3, и выполняется следующее равенство:

$$
\angle A N D=\angle B M D=90^{\circ} .
$$

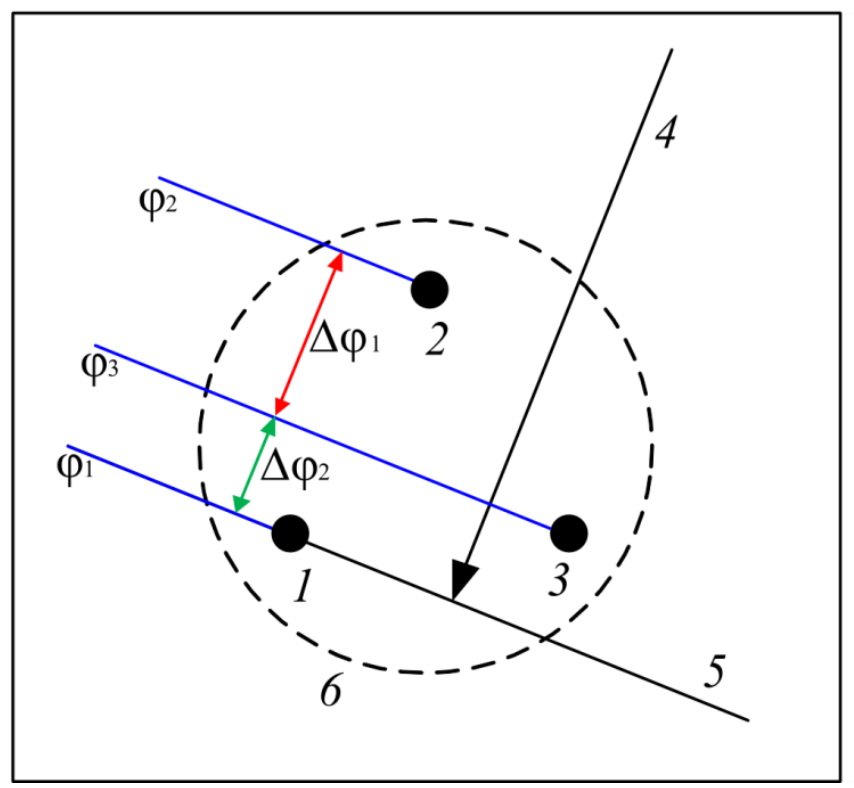

Рис. 1. Схема прохождения акустической волны через модель преобразователя:

1, 2, 3 - пьезоэлектрические преобразователи; 4 - направление волны; 5 - фронт волны; 6 - корпус датчика; $\varphi_{1}, \varphi_{2}, \varphi_{3}-$ фаза зарегистрированной волны; $\Delta \varphi_{1}, \Delta \varphi_{2}-$ разность фаз

Треугольники $\triangle A N D$ и $\triangle B M D$ подобны по первому признаку теоремы о подобии треугольников, так как $\angle A D N=\angle B D M-$ стороны одного угла являются дополнительными 
лучами сторон другого, $\angle A N D=\angle B M D$ из равенства (1). В подобных треугольниках выполняется условие пропорциональности соответственных линейных элементов и в частности:

$$
\frac{A N}{B M}=\frac{A D}{B D}
$$

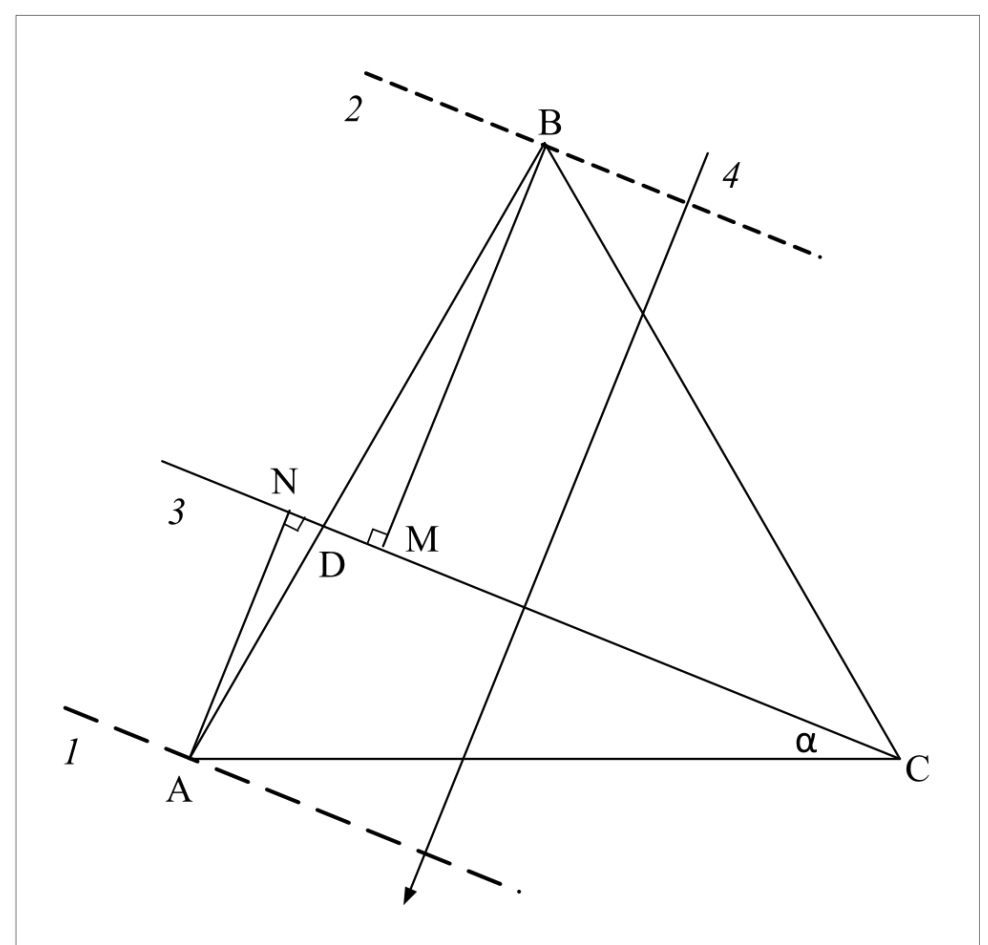

Рис. 2. Схема расстояний между фронтами волны: 1, 2, 3 - фронты волны, зарегистрированной на ПЭП 1, ПЭП 2, ПЭП 3 соответственно; 4 - направление распространения волны; пьезоэлектрические преобразователи: $A-1, B-2, C-3$

После преобразований, основанных на теореме синусов для рассмотренных выше треугольников, было получено окончательное выражение направления распространения акустико-эмиссионного воздействия:

$$
\alpha=N A C=90^{\circ}-\operatorname{arcctg}\left(\frac{2}{\sqrt{3}}\left(\frac{\Delta \varphi_{1}}{\Delta \varphi_{2}}+\frac{1}{2}\right)\right)+\pi \cdot n .
$$

\section{3. Постановка эксперимента}

Для проверки результатов решения обратной задачи проведены эксперименты на стальном листе размерами $500 \times 500 \times 8$ мм из стали Ст3пс. Акустико-эмиссионные импульсы возбуждались источником Су-Нильсена (поз. 3 рис. 3) и регистрировались тремя преобразователями акустической эмиссии типа ПП 0,1-0,7, установленными в центр листа. Основная частота регистрируемых сигналов составляла $(125 \pm 5)$ кГц и соответствующая ей длина волны для моды волны при фазовой скорости $(5,2 \pm 0,2)$ мм/мкс составляла 41 мм. Расстояние между центрами преобразователей составляло 25 мм, что удовлетворяло условиям моделирования.

Для регистрации сигналов акустической эмиссии использовалась цифровая акустикоэмиссионная система СЦАД 16.03 (ФГУП «СибНИА им. С.А. Чаплыгина», СГУПС, Новосибирск) с дискретностью отсчета аналого-цифрового преобразователя 0,5 мкс. 
Все углы отсчитывались относительно направления АC (рис. 2). Во время проведения эксперимента направление распространения акустико-эмиссионного воздействия относительно прямой, соединяющей центры преобразователей, составило $\alpha=(70 \pm 5)^{\circ}$.

Для определения степени подобия исследуемых сигналов с целью выявления фазовой задержки использовалась формула корреляции Пирсона:

$$
r=\frac{\sum_{i=1}^{n}\left(x_{i}-\bar{x}\right)\left(y_{i-\Delta n}-\bar{y}\right)}{\sqrt{\sum_{i=1}^{n}\left(x_{i}-\bar{x}\right)^{2}\left(y_{i-\Delta n}-\bar{y}\right)^{2}}},
$$

где $x_{i}$ и $y_{i}$ - сигналы акустической эмиссии с номером измерения АЦП $i ; \Delta n-$ сдвиг сигнала $x$ относительно $y ; n$ - количество измерений на рассматриваемом интервале.

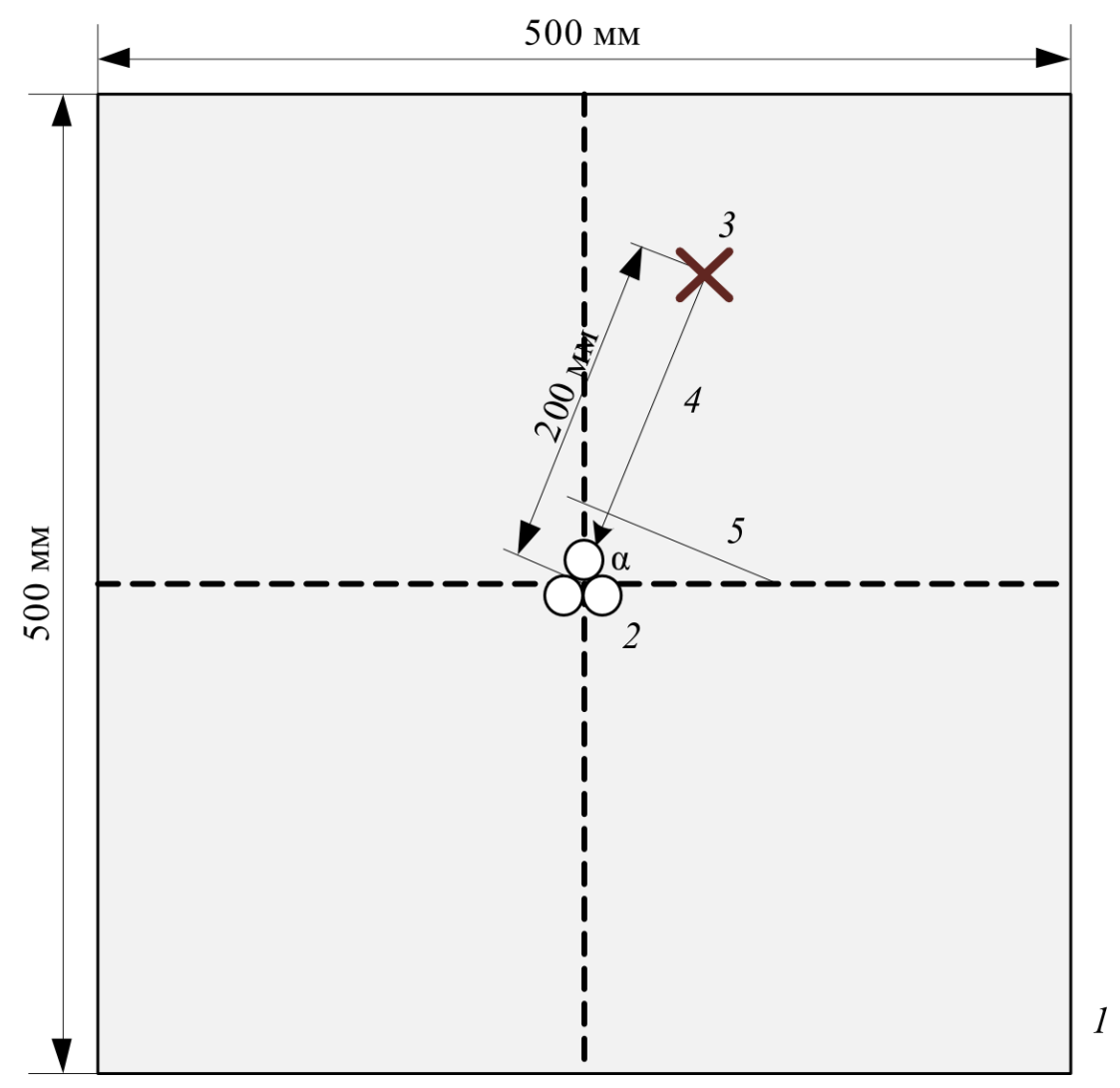

Рис. 3. Схема эксперимента: 1 - контролируемый объект стальной лист толщиной 8 мм; 2 - близкорасположенные преобразователи акустической эмиссии; 3 - место возбуждения события акустической эмиссии; 4 - направления распространения волны; 5 - фронт волны

Как видно из рис. 4, сигналы акустической эмиссии на соседних датчиках похожи по своей форме, но сдвинуты по фазе. Добавив поправку на разность фаз сигналов, можно добиться высокой степени подобия исследуемых сигналов на ограниченном интервале сравнения. Критерием степени подобия служит коэффициент корреляции (4). Коэффициент $\Delta n$ соответствует сдвигу интервалов сравнения сигналов с преобразователей и принят как разность фаз $\Delta \varphi$ в пределах рассматриваемого интервала. При $\Delta \varphi_{1}=10 \cdot 2 \pi / \mathrm{T}$ рад и $\Delta \varphi_{2}=4 \cdot 2 \pi /$ Т рад коэффициенты корреляции достигли максимальных значений на рассматриваемых интервалах и составили $r_{1}=0,93$ и $r_{2}=0,87$ соответственно, что указывает на подобия исследуемых сигналов. Расчетный угол получен подстановкой значений $\Delta \varphi_{1}$ и $\Delta \varphi_{2}$ в формулу (3) и составил $\alpha=73,5^{\circ}$. 
http://dream-journal.or"n
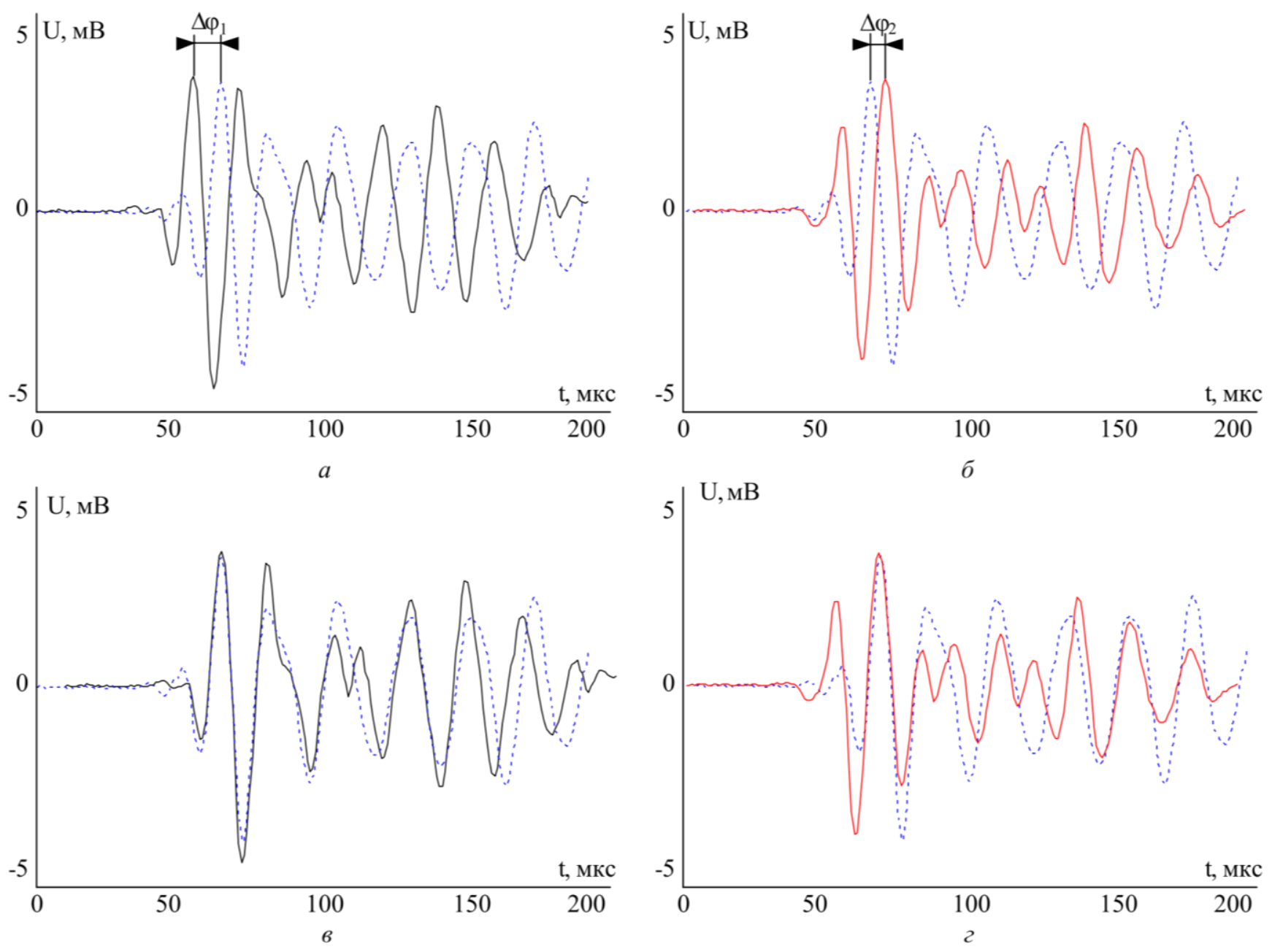

Сигнал акустической эмиссии с ПЭП №1

Сигнал акустической эмиссии с ПЭП №2

Сигнал акустической эмиссии с ПЭП №3

Рис. 4. Графики зависимости амплитуды сигналов от времени с поправкой на фазовую задержку $(\sigma, 2)$ и без нее $(a, b)$ между преобразователями 2 и $3(a, \sigma), 1$ и $3(6,2)$

\section{4. Заключение}

Для рассчитанной модели фазовой локации с использованием близкорасположенных преобразователей, направление распространения акустико-эмиссионного воздействия пропорционально отношению разности фаз между преобразователями и не зависит от скорости распространения и частоты волны.

Применение фазовой локации источников акустической эмиссии близкорасположенными относительно длины волны преобразователями позволяет определить направления источника акустической эмиссии. В результате экспериментальных исследований для разработанной модели удалось определить направление источника акустической эмиссии с погрешностью в $3,5^{\circ}$.

\section{Литература}

1. Неразрушающий контроль. Справочник : в 8 т. / под общ. ред. В. В. Клюева. Т. 7 : в 2 кн. Кн. 1. В. И. Иванов, И. Э. Власов. Метод акустической эмиссии. Кн. 2. Вибродиагностика / Ф. Я. Балицкий, А. В. Барков, Н. А. Баркова и др. - 2-е изд., испр. - М. : Машиностроение, 2006. - 829 с.: ил. 
2. Акустико-эмиссионный контроль качества сварного шва в процессе остывания / Л. Н. Степанова, Е. Ю. Лебедев, С. И. Кабанов, К. В. Канифадин, И. С. Рамазанов, С. А. Бехер // Контроль. Диагностика. - 2009. - № 3. - С. 61-67.

3. Степанова Л. Н., Бехер С. А., Тенитилов Е. С. Методика определения координат дефектов при акустико-эмиссионном контроле свободных колец подшипников // Контроль. Диагностика. - 2010. - № 4. - С. 61-65.

4. Murav'ev V. V., Bekher S. A., Vlasov K. V. Analysis of Errors in Determining the Coordinates of Acoustic-Emission Sources in Sheet-Steel Structures // Russian Journal of Nondestructive Testing. - 2008. - Vol. 44, iss. 7. - P. 480-485. - DOI: 10.1134/S106183090807005X.

5. Неразрушающий контроль. Справочник : в 8 т. / под общ. ред. В. В. Клюева. Т. 3 : И. Н. Ермолов, Ю. В. Ланге. Ультразвуковой контроль. - 2-е изд., испр. М. : Машиностроение, 2006. - 864 с.: ил. 